\title{
SISTEMATIZACIÓN DE UNA EXPERIENCIA, EVALUANDO PARA MEJORAR. GESTIÓN DE PROYECTOS
}

\author{
Guillermo Aguilar Mata* \\ Evelyn Chen Quesada**
}

El presente artículo ofrece varios elementos de carácter teórico y práctico acerca de la evaluación de proyectos que se están ejecutando. La reflexión se nutre de la experiencia acumulada en el trabajo universitario. En el marco de un modelo flexible de evaluación se sugieren una serie de dimensiones de análisis asi como orientaciones para su operacionalización. Asimismo, se sugieren estrategias complementarias que permitan aumentar el rigor con el cual se ejecuta el proceso de evaluación, tales como el manejo de la información, las reglas establecidas entre evaluadores y evaluados, entre otras.
Analysis of several theoretical and practical elements used in project evaluation. This reflection is based on experiences within the university. A series of dimensions of analysis and guidelines for their operation are offered within the framework of a flexible evaluation model. Also, additional strategies are suggested in order to increase project evaluation precision. Some of the strategies refer to information management and rules to be followed by evaluators and those being evaluated.

* Académico de la Dirección de Investigación, UNA, Coordinador Programa de Gestión de Proyectos, Profesor investigador Escuela de Sociología,graduado en Sociología y Maestía Gerencia de Proyectos de Desarrollo, Impartido cursos en materia de Gestión y Evaluación de proyectos sociales.

** Académica de la División de Educación para el Trabajo, CIDE, UNA. Graduada en Educación General Básica, Problemas de Aprendizaje, Administración Educativa y Maestría en Mediación y Conciliación en Resolución Alternativa de Conflictos. Ha impartido más de cursos en materia de investigación, evaluación, planificación, curriculum, etc. Actualmente labora como Asesora de Cooperación Internacional en la UNA. 


\section{Presentación}

El artículo es el fruto de una reflexión de equipo que tuvo lugar apartir de una experiencia multidisciplinaria, en la cual académicos provenientes de la sociología, la economía, las ciencias de la educación y el trabajo social, enfrentaron la tarea de evaluar un proyecto como parte de sus tareas de pares externos.

El grupo de profesionales mencionados conformó una comisión evaluadora la cual estableció los lineamientos básicos que acompañarían este proceso y definió las pautas por donde transitaría la evaluación, así como el modelo evaluativo.

Es importante citar que el proceso de evaluación se realizó teniendo como foco de atención la calidad en la ejecución del proyecto, el desempeño de quienes lo ejecutaron, sus carencias y las virtudes en su desarrollo.

El retomar dicha experiencia permitió valorar y ubicar con mayor rigor el papel que desempeñaron las diferentes instancias de la Universidad, responsables de la concreción y ejecución del proyecto.

Además, se hace referencia a aspectos que a nuestro juicio son importantes, en tanto apuntan u orientan sobre aspectos metodológicos, los cuales permiten detectar fortalezas y debilidades de los proyectos. Por lo tanto, el objetivo de este artículo es dar a conocer orientaciones, no solo a especialistas en el tema, sino también a ejecutores de proyectos, orientaciones que se pueden tomar en consideración para garantizar la buena marcha de los proyectos y por ende un mejor aprovechamiento de los recursos, esto en el marco de una estrategia de mejoramiento continuo y de construcción de la calidad.

Por último, el documento pretende contribuir a que los gestores de proyectos $\mathrm{y}$, en general, a que los profesionales que los tienen bajo su responsabilidad, debatan sobre el tema y además tengan a la mano la reflexión sobre una experiencia profundamente aleccionadora vivida por una comunidad científico académica.

\section{El por qué de la evaluación en los proyectos}

La evaluación de un proyecto forma parte de un proceso institucional que debe serejecutado en diferentes niveles, con el propósito de concretar un proceso dirigido al mejoramiento continuo para la construcción de la excelencia universitaria. Una acción de este tipo requiere del compromiso de diferentes actores institucionales, tales como la coordinación del proyecto, los miembros del equipo ejecutor, la comunidad académica y las autoridades de las instancias de decisión superior. En los casos de que la acción incorpore la cooperación internacional, se sumarán al proceso los pares evaluadores externos en tomo. La participación de estos actores proporciona un valor agregado a la evaluación entorno a lo científico y lo académico desde la perspectiva 
de la complementariedad de capacidades. Pero, además, permite tener una visión del estado de la cuestión más integral y multidimensional, y por ende crecientemente objetiva, garantía mayor para la toma de decisiones acertadas y pertinentes y con impacto en los procesos de vinculación de la universidad con la sociedad ${ }^{1}$.

En términos estratégicos, para el logro de un proceso de evaluación como el que se implementa se requiere, en primera instancia, que los académicos conozcan con precisión o al menos hayan participado en la construcción de:

- Las dimensiones, variables, e indicadores del modelo evaluativo

- Los parámetros y criterios ${ }^{2}$ de calidad (pertinencia, eficiencia, eficacia, factibilidad e impacto) que se van a utilizar en los procesos evaluativos a lo largo de la vida del proyecto.

- Las especificaciones metodológicas y los mecanismos definidos con anterioridad. Estas especificaciones van desde el análisis conjunto de la estrategia de evaluación hasta los resultados obtenidos.

- El modelo evaluativo y los instrumentos o técnicas de evaluación deben estar contextualizados y conceptualizados en función de las particularidades y la historia de vida que configuran la naturaleza de los proyectos.

Las evaluaciones no son un fin en sí mismo, sino que se constituyen en mecanismos por medio de los cuales se puede variar el curso de un proyecto cuando se desarrollan mejores políticas, estrategias, programas y de paso obtener mejores resultados.

Por otra parte, el ejercicio de la evaluación puede adquirir diferentes modalidades, las cuales están en alguna medida determinadas por el lugar del proyecto en su ciclo de desarrollo. Por ejemplo, si el proyecto ya ha finalizado podemos evaluar su impacto, si el proyecto se está formulando se puede evaluar su coherencia y así sucesivamente. Ahora bien, si el proyecto está en ejecución, con resultados en camino, con una historia de vida caracterizada por el conflicto y la resolución de ellos, la evaluación debe centrarse en los procesos, en el rabajo del equipo, en el sistema de relaciones, sus liderazgos y en los resultados de esta ecuación. Esta opción metodológica retoma algunos elementos y aportes de la evaluación del desempeño ${ }^{3}$ y oras teorías que nos obligan a pensar en el objeto por evaluar como un sistema completo, complejo, coherente e interrelacionado. Lo anterior significa que los resultados de la evaluación no solo tienen la misión de realimentar y mejorar al objeto sino, también, aportar elementos para

Nirenberg Olga, et al, Evaluar para la transformación, Editorial Paidos, Buenos Aires , Argentina,2000.

Ver cuadro 1: Criterios de evaluación.

La evaluación del desempeño se ubica en una escuela de pensamiento que ya tiene fuertes críticos, tales como Tom

Coens en su libro $i$ Evaluaciones de desempeño? Por que no funcionan y como reemplazarlas (2001). 
mejorar su sistema de relaciones con el entorno. Esto permite que los actores, ya sea de forma individual o grupal se comprometan con los resultados de la evaluación para lograr un máximo aprovechamiento de la esta.

Si bien es cierto el análisis sistémico que proponemos significa enfrentar el trabajo en múltiples dimensiones, es necesario identificar aquellas que para el proceso son estratégicas, lo cual tiene que ver con la praxis desarrollada por el proyecto, el grupo humano ejecutor y el sistema de relaciones con el entorno. En síntesis, una definición a priori de ellas no es conveniente; conocer el proyecto en todas sus dimensiones es un imperativo categórico.

Según nuestra experiencia en la evaluación de diferentes proyectos, se consideran al menos los siguientes aspectos:

1. El contexto.

2. El sistema de relaciones entre las partes y contrapartes.

3. Los procesos desarrollados.

4. Las principales líneas de acción.

5. Aspectos a considerar en el proceso de evaluación:

- Definición de estrategia.

- Socialización y negociación de estrategia seleccionada y acuerdos.

- Construcción del modelo evaluativo, instrumentos y procedimientos.

- Programación del plan evaluativo.

- Cuidados con el manejo de la información.

- Socialización de los resultados obtenidos.

- Involucramiento de los actores en la toma de decisiones.

- Metaevaluación.

\section{El contexto donde se desarrolla el proyecto}

En esta dimensión, el análisis se debe centrar en la valoración de la formalización o institucionalización del proyecto al interior de la organización en sus diferentes instancias, tales como Rectoría, Vicerrectorías, Facultad o Centro, Unidad Académica, Departamentos; y si cuenta con apoyo externo, ya sea nacional o internacional es necesario valorarlo.

En esta dimensión se consideran los insumos para asumir la ejecución del proyecto. Es decir, insumos como los recursos financieros, los financieros y la infraestructura. Además de contar con todo lo anterior, es necesario demostrar la existencia de equipos humanos con capacidad de desarrollo de conocimiento insertado (es decir, conocimiento que reside fundamentalmente en las relaciones especializadas entre individuos y grupos y en las particulares normas, actitudes, 
flujos de información y forma de tomar decisiones que caracterizan los contactos entre ellos ${ }^{4}$ ), con experiencia en líneas de trabajo o de investigación, docencia, extensión y producción la cual se ha expresado en resultados tangibles como:

- Proyectos ejecutados con niveles de éxito y calidad demostrados según los parámetros internacionales para el sector de conocimiento.

- Fondos externos captados y utilizados eficientemente.

- Proyectos que han desarrollado capacidad social, es decir, el logro de impactos sociales como producto de su acción sistemáticamente orientada.

- Proyectos innovadores en docencia que hayan demostrado su capacidad para ser replicados en otros ámbitos académicos, con un alto nivel de efectividad.

En otras palabras, el contexto donde se originan los proyectos debe tener bases sólidas, sustentadas en una realidad institucional comprometida con el quehacer que implica el desarrollo e implementación del proyecto.

El conocimiento y la aprehensión de esta dimensión es garantía inicial para identificar el conjunto de determinantes que configuran, orientan y en última instancia pueden definir el éxito o fracaso de un proyecto.

\section{El sistema de relaciones entre las partes y contrapartes}

Con respecto a este punto, consideramos importante tener claridad sobre la estructuración del conjunto de relaciones de poder que entre las personas, los equipos de trabajo y con el entorno, se hilvanan y se desarrollan para la ejecución del proyecto.

Entre ellas, hay relaciones de jerarquía, fruto de una estructura ins itucional, relaciones científico-académicas que están en la base de los procesos de producción de conocimiento, las relaciones de coordinación y cooperación que se construyen, legitiman y expanden las fronteras internas y externas del proyecto, brindando la posibilidad para la internacionalización del conocimiento.

Todas estas relaciones deben estar finalmente enlazadas de tal manera que permitan, el desarrollo de las relaciones interpersonales, el crecimiento profesional, el intercambio de experiencias, el fortalecimiento de acciones futuras, la claridad en el seguimiento y evaluación, todo para construir un clima organizacional óptimo para el buen desempeño del proyecto.

Las relaciones no se pueden abstraer de los actores, estos son portadores de una historia personal y académica, de intereses que obedecen a su ubicación estructural, a su desempeño coyuntural y al resultado de alianzas entre ellos.

Badaraccco, Joseph L., Alianzas estratégicas y el caso de la General Motors e IBM. McGraw-Hill, México, 1992. 
La identificación de estos intereses se convierte en un material heurístico para entender el carácter que las relaciones asumen durante la vida del proyecto y le dan su especificidad. Esto aún toma más importancia cuando las relaciones dejan de ser acciones individuales para convertirse en acciones conjuntas, determinadas por una buena comunicación y negociación en la toma de decisiones.

\section{Los procesos desarrollados en el marco de la organización del proyecto}

En este contexto es necesario reivindicar la importancia de observar y valorar los procesos que se despliegan para la consecución de los objetivos del proyecto. Tradicionalmente, las evaluaciones no los han considerado, porque se ha brindando exclusivamente atención a los resultados y, en particular, a los impactos. Tomar en consideración la evaluación de los diferentes procesos permitiría a los responsables generar información valiosa para la toma de decisiones relacionadas con ajustes o cambios en materia de presupuesto, recursos humanos, metas, objetivos o resultados.

Otra ventaja de trabajar en evaluaciones de proceso, durante la ejecución del proyecto, es que se puede visualizar y fortalecer la sostenibilidad del proyecto, la creación de nuevas líneas de acción y ejecución, la integración de conocimientos y la definición y redefinición de estrategias metodológicas.

\section{Aspectos por considerar en un proceso de evaluación de proyectos}

En la actualidad se han desarrollado una gran variedad de metodologías y estrategias que han sido diseñadas para la evaluación de proyectos. Considerando la evaluación como un proceso, es interés de este artículo referirse a un conjunto de etapas que pueden ser tomadas en consideración por los expertos en las diferentes áreas de trabajo académico universitario. A continuación se presentan los aspectos que tienen que tomarse en cuenta.

\section{Definición de estrategia de evaluación}

Dada la definición que hemos ido construyendo de evaluación como un proceso y no como un hecho aislado, la estrategia de evaluación debe estar predeterminada desde la formulación misma del proyecto, momento en el cual se identifican los indicadores de éxito del proyecto que deben ser conseguidos a lo largo de su ejecución. A esta definición inicial de estrategia de evaluación le debe ser inherente contar con una relación entre esta dimensión micro del proceso académico, con una dimensión meso, es decir, las políticas y estrategias institucionales de evaluación, y la dimensión macro que da cuenta del modelo de calidad que la institución ha adoptado. 
Supuesto lo anterior, el proceso de evaluación cuenta con parámetros de excelencia que lo orientan, a partir de los cuales se construye la estrategia particular.

Esta estrategia de evaluación debe establecer con claridad los sujetos de la evaluación, tales como el proyecto en general, el equipo ejecutor y otros que acompañarán el proceso.

La formulación de la estrategia debe, además, contemplar las especificaciones metodológicas, propuestas tanto por parte de los evaluadores internos como externos al proyecto. De lo anterior se desprende que, para llevar a cabo la conformación y el desarrollo del trabajo de una comisión o comisiones de evaluación, toman especial importancia los objetivos del proceso, las metas, el diseño de los instrumentos, los momentos de consulta y otros aspectos.

En la construcción de dicha estrategia, el equipo evaluador o comisión debe dedicar tiempo para sesiones de trabajo destinadas a identificar las particularidades del proyecto, para así responder con una estrategia específica y garantizar una estructura básica que ordene el proceso.

A continuación se presenta un esquema que resume algunos aspectos por contemplar en la estrategia:

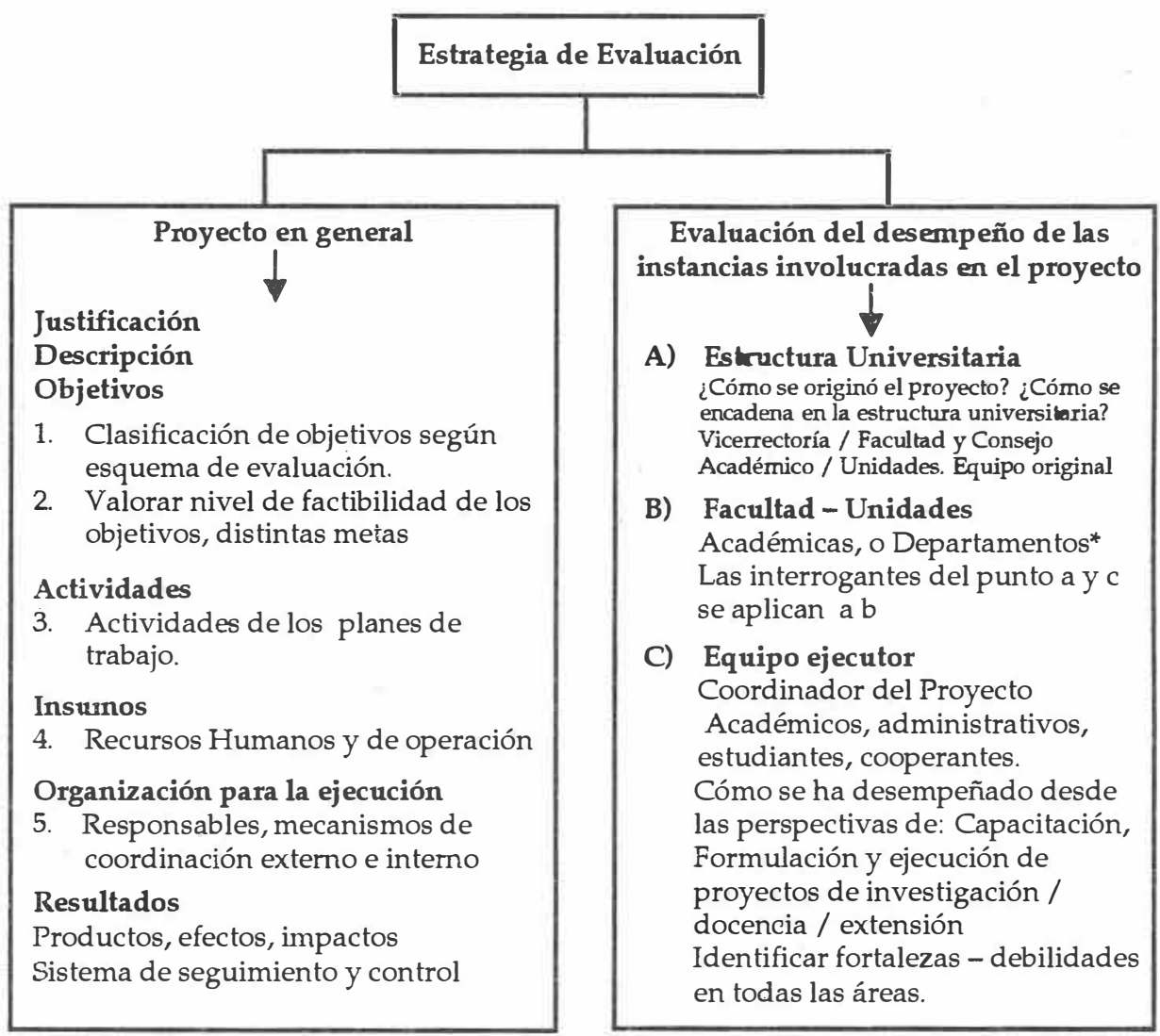


En el esquema anterior se enumeran algunos componentes que se consideran en el diseño de un proyecto. La estrategia de evaluación, deberá permitir la identificación y verificación sobre cuánto de lo programado se está cumpliendo en los téminos previamente definidos de cantidad y calidad definidos. Para lograr lo anterior, el equipo evaluador debe tener acceso a la documentación y, en general, a la información que da fe de los resultados obtenidos, de la contribución de la organización, de lo pertinente y oportuno de los insumos, de la calidad de las actividades y del logro de los objetivos.

A continuación se brinda una breve explicación de los elementos que se consideran oportunos de evaluar en esta sección y que: la justificación, la descripción, los objetivos y metas, las actividades, los insumos, la organización para la ejecución, los resultados y el sistema de seguimiento y control.

\section{Justificación}

Se debe evaluar los antecedentes que motivaron el proyecto. En ella se describe tanto la situación actual como la posición de la organización antes de realizarlo.

Además es importante indagar sobre los argumentos que se esgrimieron para demostrar la pertinencia del proyecto, así como los posibles impactos.

Por último, es recomendable valorar los diferentes escenarios que se describieron como parte de la organización del proyecto, una vez alcanzados los resultados esperados.

\section{Descripción}

Según José Luis Sollerio (1989), en el “Módulo de Diseño y la Gestación del Proyecto de Innovación", se distinguen dos vertientes principales en la descripción de los proyectos. La primera tiene que ver con la planeación y metodología empleada para su desarrollo; la segunda, con la descripción de las actividades. Por tanto, en el proceso de evaluación será de interés verificar si la descripción es concordante con la integralidad del proyecto, es decir, con su secuencia lógica para la realización de su planificación en tomo a los recursos financieros, humanos, equipamiento, etc., y la descripción con precisión de sus alcances y articulación.

\section{Objetivos y metas}

Solo aquellos proyectos que presenten objetivos claros, precisos, mensurables y factibles son sujetos de aprobación. El objetivo debe interpretar adecuadamente 
la necesidad u oportunidad que tratará de atender. Además de revisar los objetivos en térnninos de claridad y precisión, es importante tener en cuenta la viabilidad de alcanzarlos. ${ }^{5}$

\section{Actividades}

Las actividades deben responder de forma directa a los objetivos planteados en el proyecto. La evaluación de ellas impone la necesidad de claridad y objetividad, ya que de las mismas se derivan los recursos requeridos por el proyecto.

\section{Insumos}

Generalmente la asignación de recursos al proyecto está condicionada por su objetivo. En la evaluación de los insumos requeridos por el proyecto hay que observar la importancia de los especialistas asesores y equipos mayores requeridos por algunas actividades, que frecuentemente no son de una disponibilidad inmediata. Al respecto, Luis Sollerio (1989) señala que para el caso de las actividades internas, es indispensable el buen desempeño del rol del padrino, para comprometer a la dirección de la organización con el proyecto. Se requiere que exista una buena coordinación con las áreas operativas, a fin de permitir la realización de actividades relacionadas con el proyecto.

\section{Organización para la ejecución}

En este apartado es importante constatar el grado de participación que han tenido tanto los responsables directos como indirectos del proyecto. Asimismo, los mecanismos de coordinación que se han implementado entre los diferentes equipos de abajo que constituyen el proyecto, así como la organización utilizada para la relación con el entomo.

\section{Resultados}

La evaluación de los resultados (productos, efectos e impactos) estará mediada por logro de los objetivos así como de la viabilidad y factibilidad de cada uno de los aspectos que conforman el proyecto. Es importante considerar la naturaleza de los resultados, por que no siempre son tangibles, lo que sugiere un esfuerzo adicional para describir los beneficios indirectos, intangibles o subjetivos.

5 Centro Universitario de Desarrollo. (1989). Gestión de un Proyecto. Programa de Desarrollo de las Naciones Unidas, Secretaría del Convenio de Andrés Bello (SECAB). Serie de Manuales de I\&D (7). 
El sistema de control y seguimiento del proyecto está íntimamente relacionado con la planeación y la descripción de actividades realizadas. Un buen sistema para este efecto será el que nos proporcione información expedita sobre las actividades, tanto las concluidas como las pendientes. Asimismo, debe integrar la información correspondiente a los plazos y los recursos consumidos y aquellos que se requieran en un período determinado.

Por lo que respecta a las funciones de control, la información recopilada por el sistema de seguimiento apoyará el proceso de toma de decisiones que determinará las acciones necesarias para corregir las desviaciones de los planes originales. ${ }^{6}$ En otros términos, se constará si el proyecto cuenta con un sistema de seguimiento y control, identificando los resultados obtenidos Por último es prudente determinar si la información sistematizada ha sido utilizada y de qué manera lo ha sido.

\section{Evaluación del desempeño de las instancias involucradas en el proyecto}

Así mismo el esquema (cuadro 1) plantea la necesidad de valorar la formalización del proyecto en la estructura universitaria, en sus diferentes instancias y el funcionamiento del mismo bajo las figuras de conducción superior de la organización,( como pueden ser Rectoría, el nivel de facultad, el de unidades académicas), todas ellas, que participan en la aprobación y ejecución del proyecto. Teniendo en mente dicho objetivo se hace necesario valorar la participación, el grado de involucramiento y los niveles de responsabilidad de los actores en dicho proceso de formalización.

En este sentido, se observa la figura del equipo ejecutor, la cual identifica las funciones tal como están definidas jerárquicamente, ya que estas son la base de las relaciones de poder, y que de una u otra manera dejan su impronta en las relaciones interpersonales que se establecen al interior del equipo y de este con el entorno.

De lo anterior se desprende cuáles aspectos como liderazgo en la gestión en su política y estrategia, en los recursos, en la gestión del proceso y en sus resultados son parte de los criterios que se deben contemplar en la construcción de la estrategia?.

6 Centro Universitario de Desarrollo. (1989). Gestión de un Proyecto. Programa de Desarrollo de las Naciones Unidas. Secretaría del Convenio de Andrés Bello (SECAB). Serie de Manuales de I\&D (7).

7 Método parta mejorar la calidad de la Educación Superior Basado en el Modelo del EFQM, Productos, Club Gestión de la Calidad ,1999. 
Es imperativo tener presente en esta visión que la evaluación del desempeño de la coordinación tiene un impacto importante en la dinámica de grupo. Ambos influyen en la vida del proyecto, en la forma como se pretende alcanzar las metas intermedias y finales, el estilo de gestión para la toma de decisiones, la asignación de las tareas y las responsabilidades, así como la definición del perfil del equipo y de los perfiles particulares de los miembros de este.

El papel que desempeña el directivo o coordinador de un proyecto es de carácter fundamental para el logro de los objetivos de un plan de trabajo, especialmente cuando esto implica la participación de un equipo de trabajo conformado de manera interdisciplinaria.

Por otra parte, los trabajos realizados recientemente acerca de la dirección eficaz de equipos, coinciden en que existen dos tipos de comportamiento en los directivos:

- Los comportamientos relacionados con la mejora de la motivación y la ilusión de pertenecer al equipo se denominan Comportamientos de Apoyo. Son comportamientos que permiten un mantenimiento del equipo y se orientan, sobre todo, a potenciar aspectos de relación, comunicación e interacción entre los miembros, centrados en el desarrollo de la armonía del equipo. Prestan especial atención a cómo está funcionando el equipo (incluye temas como dar reconocimiento, armonizar, mediar en conflictos, compartir, crear relaciones, etc.)

- Los comportamientos que afectan de forma directa a la competencia del equipo se denominan Comportamientos de Tarea. Son aquellos comportamientos orientados a cómo hacer las cosas y prestan especial atención a lo que se supone que el equipo debe hacer; aportan dirección al equipo (incluye temas como establecer y aclarar metas, dar instrucciones, establecer límites, dar información, organizar agendas, etc. $)^{8}$

Ambos ipos de comportamiento son fundamentales si el equipo desea desamollarse completamente, y conviene que sean compartidos por todos los miembros.

En cuanto al manejo de conflictos por parte de la coordinación o administración de un proyecto tiene una importancia fundamental para el logro de equipos de trabajo eficaces, con el propósito de conciliar o mediar las dificultades naturales por las que atravesarán los integrantes durante la planificación, desarrollo, ejecución y evaluación de proyectos.

Para impulsar el proceso de desarrollo de un equipo, la dirección del proyecto debe conocer su comportamiento como líder, según las necesidades del equipo.

Saber que existen variaciones en los niveles de competencia y de motivación de los equipos, que son previsibles de una fase de desarrollo a otra, y que

8 Fundamentos de trabajo en equipo: equipos eficaces, pág. 26, 2001. 
La evaluación de un proyecto

forma parte de un proceso institucional que debe ser ejecutado en diferentes niveles con el propósito de concretar un proceso dirigido al mejoramiento continuo para la construcción de la excelencia universitaria. Una acción de este tipo requiere del compromiso de diferentes actores institucionales, tales como la coordinación del proyecto, los miembros del equipo, la comunidad académica y las autoridades de las instancias de decisión superior. los equipos no van a alcanzar todo su potencial hasta que atraviesen todas las fases y llegar a la producción, tiene muchas implicaciones de cara a los líderes y miembros del equipo. No solo explica lo que es probable que pase en determinada fase de la vida del equipo, sino lo que es más importante, sugiere que la principal función del directivo de un equipo es centrarse en ayudarlo a medida que este avanza a lo largo de las fases de desarrollo.

En conclusión, la estrategia permite, entre otras cosas, detectar los logros más importantes, así como las dificultades u obstáculos que enfrentan los proyectos desde sus orígenes. A partir de este proceso de valoración, el equipo evaluador empieza acumular un conocimiento que va a permitir

medir la calidad y la eficiencia en la intervención propuesta por el proyecto.

Establecida la estrategia, queda, entre otras cosas, organizar el tipo y cantidad de entrevistas (estructurada, abierta, etc.), así como el número de personas y roles que desempeñan. Esto en sesiones con duración suficiente para abarcar los temas que se han definido como fundamentales y de esta manera poder sistematizar la información y proceder a su sistematización de manera efectiva.

Como parte de este momento, contemplar visitas al campo donde se ejecutan las actividades del proyecto, complementa y verifica la información obtenida en las entrevistas a los diferentes actores que participan en la ejecución del proyecto o en su aprobación.

Por último, estructurada la estrategia de evaluación, el equipo evaluador deberá darse a la tarea de socializarla y negociarla con las instancias responsables en la toma de decisiones de la organización o institución y del proyecto, esto con el fin de determinar la viabilidad y la factibilidad de ponerla en práctica. 
En términos conceptuales este momento sirve no solo para comunicar o adecuar la propuesta y para firmar los términos últimos de referencia para proceder al proceso. También sirve para afirmar la viabilidad política de las acciones por emprender y además definir las competencias y obligaciones del equipo evaluador.

Finalmente, las autoridades pueden in troducir otros elementos al proceso ya sea adoptando una opción de evaluación por pares o incorporando una visión de autoevaluación por parte del equipo ejecutor del proyecto o combinarlas, ya que son complementarias.

Como todo proceso efectivo de negociación, este debe ser objetivo, donde se involucren todas las partes participantes; flexible, que permita realizar aquellos cambios según las necesidades del proyecto; diverso, que facilite la variedad de alternativas de evaluación; comunicativo, que proponga formas o mecanismos de manejo de la información y satisfactorio, que genere niveles de ganancia para todos.

\section{Construcción de instrumentos y procedimientos}

Una vez negociado y consensuado el modelo evaluativo se hace necesario determinar las diferentes técnicas de evaluación que se van a utilizar durante el proceso. Dicho sea de paso, el modelo, de alguna manera, condiciona el tipo de técnicas elegir, las cuales deben permitir recuperar información, ya sea cualitativa o cuantitativa, o de ambos tipos.

\section{Programación de plan evaluativo}

La programación del plan evaluativo, que lleva implícita la aplicación del modelo evaluativo y la diseminación de los resultados, debe contemplar ciertos momentos, los cuales se enumeran seguidamente:

- Identificación plena de los actores que participarán en el proceso de evaluación, así como de las audiencias a los cuales serán destinados los resultados del proceso.

- La transferencia del modelo y ajustes, que se desarrolla en el marco de un proceso de capacitación.

- Definición de los momentos de recolección de información, sistematización, análisis y elaboración de conclusiones.

- Definición del momento y la estrategia de devolución de los hallazgos de la evaluación.

- Determinación de los componentes del informe de evaluación. 
En este apartado interesa llamar la atención sobre la necesidad del evaluador de definir los tipos de información que se va a solicitar y los cuidados en su manejo. En este sentido, algunas de las fuentes de información escritas que se deben tener a disposición son entre ellas: el documento de formulación o diseño del proyecto, evaluaciones parciales (si existen), informes de avance, o de productos, acuerdos internos o internacionales si los han requerido, planes de trabajo y sus respectivos presupuestos, así como cualquier otro documento que facilite una mayor información sobre lo evaluado o lo que se va a evaluar.

También existen otros tipos de fuentes de información tales como los sujetos participantes, los grupos meta, técnicos, asesores, consultores y otros que sean necesarios.

Con respecto al manejo de la información es necesario definir en la estrategia de evaluación cuáles van a ser los cuidados que se van a tener durante el acceso a la misma, esto requiere tomar decisiones que pueden implicar connotaciones éticas, morales y legales; un ejemplo de ello es determinar con anterioridad si los informes protegerán a los informantes de futuros conflictos o implicaciones laborales, etc. Todo ello dependerá de los objetivos que persigue la evaluación. Con base en lo anterior, la confidencialidad es una condición que desarrolla, en una gran mayoría de los informantes, confianza y seguridad por brindar información al respecto de un asunto, pero esto de igual manera también se tendrá que definir en la estrategia.

Otro aspecto que los evaluadores deben considerar, y al que no es común dedicarle tiempo y pensamiento, es la preparación de las sesiones de trabajo con sujetos informantes del proyecto, lo cual se puede visualizar en dos momentos. El primero tiene que ver con la preparación a priori de los evaluadores con respecto a la forma en que se van a desempeñar durante el proceso de las sesiones evaluativas. Es decir, tener claro quién va a conducir la reunión, cuáles son las preguntas clave, los roles y los momentos de participación. No es conveniente lanzar una batería muy amplia de preguntas al mismo tiempo, ya que por la necesidad de abarcarlas todas se puede perder información, o que a su vez, los evaluadores formulen preguntas al mismo tiempo sobre aspectos diferentes. Por lo tanto se recomienda desarrollar una escucha activa no solo del lenguaje verbal sino corporal y permitir que los informantes concluyan su exposición sin interrupciones De lo anterior se desprende que el evaluador es un facilitador de un proceso.

Un segundo momento, está relacionado con la reunión, para lo cual hay que considerar el espacio físico donde se realizará el proceso, el uso de tecnología apropiada (vídeo, grabadora, etc.) y las condiciones adecuadas para que los actores puedan concentrase en la actividad sin interrupciones, son algunos de los aspectos por considerar. 
En cuanto a la reunión propiamente, es conveniente dar la bienvenida, agradecer la presencia, explicar el rol del evaluador (s) su carácter de imparcialidad, así como el propósito de la reunión, los procedimientos a seguir, y las reglas que orientaran el proceso, tales como, el respeto entre las partes, la no interrupción entre los participantes, la confidencialidad, el tiempo de trabajo, la posibilidad de tener reuniones separadas con algunos de los participantes, los momentos para aclarar dudas y, por último, quién va ceder la palabra; todo lo anterior generará un clima apropiado para el logro de los objetivos de la evaluación, la cual tendrá como colofón, las conclusiones que valorarán los resultados de aquel.

\section{Socialización de resultados obtenidos}

Finalizado el proceso y obtenida la información requerida, es necesario recuperar lo planteado en el apartado de programación, anteriormente desarrollado. Se hace necesario elaborar una guía de informe y la estrategia para la socialización y legitimación de este ante los responsables del proyecto y los tomadores de decisiones de la organización, lo cual asegurará la toma de decisiones adecuadas y a tiempo, que permitan orientar o reorientar el proyecto.

Esta estrategia debe considerar a los actores y las audiencias hacia las cuales debe ir dirigida la información, ya que esto determinará la forma y el momento de la divulgación.

Por ultimo, este es el mejor momento para que el informe sea realimentado y facultado, o se aclaren aquellos aspectos que se han considerado dignos de ello. Sin embargo esto no debe ser considerado una condición sine qua non, sobre todo en los casos en que el modelo de evaluación contó con la participación de los actores, y se tomaron en cuenta los intereses de las audiencias.

\section{Metaevaluación}

En este apartado es primordial el interés de los especialistas o de los evaluadores de proyectos por considerar la metaevaluación como un medio o herramienta que permita valorar lo evaluado, tanto como lo utilizado para evaluar. Se entiende entonces la metaevaluación, según Stufflebeam 1991"...como aquella investigación sistemática cuyo objetivo es emitir un juicio acerca de la calidad o méritos de una evaluación. La evaluación se convierte de esta forma en el objeto de estudio del metaevaluador".

Según lo anterior, el evaluador puede utilizarla de acuerdo a la conveniencia para que el estudio evaluativo sea comprehensivo y comprensible, creíble, ético y que explicite los estándares de valor utilizados, así como sujustificación, factibilidad 
para ser llevado a la práctica suficiente, pero no excesivamente preciso (la evaluación ha de ser rigurosa desde un punto de vista técnico, aunque un exceso de rigurosidad puede hacer también que se olviden las cuestiones importantes.

\section{Referencia}

Autoevaluación del Proyecto "Capacitación para el Desarrollo Regional Sostenible". Período 1999-2002.

Convenio de Cooperación celebrado entre la UNA-COSTA RICA y la University of Regina. Regina, Saskatchewan, Canadá.

Descripción del Proyecto “Capacitación para el Desarrollo Regional”.

Formulación del Proyecto UNA-REGINA ante la Dirección de Docencia y Dirección de Extensión. Universidad Nacional, Campus Omar Dengo, Heredia, 1998.

Hernández, C. y Aguilar, G. Evaluación Concurrente. Revista Imágenes. Dirección de Extensión ,Universidad Nacional, Heredia, Costa Rica. 2003.

Minutas de la Comisión Evaluadora del Proyecto UNA-REGINA, de la $\mathrm{N}^{\circ}$ 01-2002 a la N 13-2002.

Mujica, M. (2001). Evaluación del progreso del Proyecto, a medio periodo. Informe Final.

Nirenberg, O. et al. (2000). Evaluar para la transformación. Innovaciones en la evaluación de programas y proyectos sociales. Buenos Aires, Argentina. Paidos,

POAI' de los años 2000, 2001 y 2002 de la Escuela de Sociología.

Pacheco, J. F. DPPI, ILPES. Métodos para resolver problemas.

Sancho, F. Documento borrador sobre Evaluación del Desempeño.

Stufflebeam(1991). Diccionario Crítico de Ciencias Sociales. Metaevalaución. 